Original paper

\title{
Feasibility of reducing differences in estimated doses in nuclear medicine between a patient-specific and a reference phantom
}

\author{
Alexandra Zvereva ${ }^{\mathrm{a}, \mathrm{b}, *}$, Helmut Schlattl ${ }^{\mathrm{a}}$, Maria Zankl ${ }^{\mathrm{a}}$, Janine Becker ${ }^{\mathrm{a}}$, Nina Petoussi-Henss ${ }^{\mathrm{a}}$, \\ Yeon Soo Yeom ${ }^{c}$, Chan Hyeong Kim ${ }^{c}$, Christoph Hoeschen ${ }^{\mathrm{d}}$, Katia Parodi ${ }^{\mathrm{b}}$ \\ ${ }^{a}$ Helmholtz Zentrum München, German Research Center for Environmental Health, Institute of Radiation Protection, Ingolstädter Landstrasse 1, 85764 Neuherberg, Germany \\ ${ }^{\mathrm{b}}$ Ludwig Maximilians Universität München (LMU Munich), Experimental Physics - Medical Physics, Am Coulombwall 1, 85748 Garching, Germany \\ ${ }^{\mathrm{c}}$ Department of Nuclear Engineering, Hanyang University, 222 Wangsimni-ro, Seongdong-gu, 04763 Seoul, Republic of Korea \\ ${ }^{\mathrm{d}}$ Otto von Guericke Universität Magdeburg, Institut für Medizintechnik, Universitätsplatz 2, 39104 Magdeburg, Germany
}

\section{A R T I C L E I N F O}

\section{Article history:}

Received 14 October 2016

Received in Revised form 4 June 2017

Accepted 6 June 2017

Available online 16 June 2017

\section{Keywords:}

\section{Nuclear medicine}

Human computational phantom

Personalised internal dose

Cross-fire

\begin{abstract}
A B S T R A C T
The feasibility of reducing the differences between patient-specific internal doses and doses estimated using reference phantoms was evaluated. Relatively simple adjustments to a polygon-surface ICRP adult male reference phantom were applied to fit selected individual dimensions using the software Rhinoceros ${ }^{\circledR} 4.0$. We tested this approach on two patient-specific phantoms: the biggest and the smallest phantoms from the Helmholtz Zentrum München library. These phantoms have unrelated anatomy and large differences in body-mass-index. Three models approximating each patient's anatomy were considered: the voxel and the polygon-surface ICRP adult male reference phantoms and the adjusted polygonsurface reference phantom. The Specific Absorbed Fractions (SAFs) for internal photon and electron sources were calculated with the Monte Carlo code EGSnrc. Employing the time-integrated activity coefficients of a radiopharmaceutical (S)-4-(3-18 F-fluoropropyl)-L-glutamic acid and the calculated SAFs, organ absorbed-dose coefficients were computed following the formalism promulgated by the Committee on Medical Internal Radiation Dose. We compared the absorbed-dose coefficients between each patient-specific phantom and other models considered with emphasis on the cross-fire component. The corresponding differences for most organs were notably lower for the adjusted reference models compared to the case when reference models were employed. Overall, the proposed approach provided reliable dose estimates for both tested patient-specific models despite the pronounced differences in their anatomy. To capture the full range of inter-individual anatomic variability more patient-specific phantoms are required. The results of this test study suggest a feasibility of estimating patient-specific doses within a relative uncertainty of $25 \%$ or less using adjusted reference models, when only simple phantom scaling is applied.
\end{abstract}

(c) 2017 Associazione Italiana di Fisica Medica. Published by Elsevier Ltd. All rights reserved.

\section{Introduction}

Radiation-based modalities are used extensively in diagnostic and therapeutic medical applications. Their usage requires the estimation of radiation doses to the patient. International Commission on Radiological Protection (ICRP) Publication 110 [1] describes the reference voxel computational phantoms that are widely used in dosimetric calculations. Xu [2] stated that for medical dose tracking individualised dosimetry is needed. This is in contrast to the

\footnotetext{
* Corresponding author at: Ingolstädter Landstrasse 1, 85764 Neuherberg, Germany.

E-mail addresses: alexandra.zvereva@helmholtz-muenchen.de, azvereva@bfs.de
} (A. Zvereva).
"Reference Man" methodology promulgated by ICRP and used in prospective radiation protection. Bolch et al. [3] mentioned the limited use of reference phantoms in medical imaging and therapy applications, because anatomical variations among individuals can notably contribute to the uncertainty of the estimated organ doses. These variations are disregarded if reference phantoms are employed. Thus, the application of individualised phantoms has the potential to improve the accuracy of medical dosimetric calculations. This is especially important for medical dose estimation in therapeutic applications. The accuracy level of dosimetry depends on the application and on the available resources. The accurate segmentation of a complete patient-specific phantom in nuclear medicine is often difficult, due to the very time-consuming manual work necessary. Another shortcoming of the segmentation of 
individual phantoms is the need of individual tomographic images of sufficient resolution and body coverage, which are usually not available in routine nuclear medicine procedures.

Up to now, many groups created families of human computational phantoms via segmentation from available individual tomographic data or via scaling BREP (Boundary REPresentation) phantoms in terms of body height and weight [4-19]. Total body height, weight or body mass index (BMI) are often used as parameters for matching a specific individual with a library of available phantoms for the purpose of dosimetric calculations. Nonetheless, this usually does not reflect the organ topology of the investigated individual and could cause large differences between the real patient-specific doses and those obtained for the available computational phantom. Zhang et al. [20] investigated the differences in organ doses, effective doses, and risk indices in computed tomography (CT) examinations for adults, caused by the employment of four types of computational phantoms in the dosimetry. This included the extended cardiac-torso (XCAT) family [21], the reference voxel phantoms described in ICRP Publication 110 [1] and two stylized mathematical phantoms. The study of Zhang et al. [20] revealed considerable differences in organ doses from CT even in case of closely matched weight, height and organ masses of the investigated phantoms. Other authors also quantified and showed cases with notable differences in individual organ dose conversion coefficients for external irradiation $[4,6]$ and Specific Absorbed Fractions (SAFs) or organ absorbeddose coefficients for internal irradiation [22-24], caused by the variations in organ masses and locations in different computational phantoms.

This work focuses on nuclear medicine diagnostic investigations. In this case, organs and tissues of a patient receive low to moderate doses due to the accumulation of an administered radiopharmaceutical in various body organs and tissues, so-called source regions. There are two components of an organ absorbeddose: (1) the dose from the self-absorption (for source regions only), (2) the dose from the cross-fire from other source regions $[25,26]$. The self-absorption dose is the dose deposited in a tissue from radiation contained in the tissue itself. The dose from the cross-fire denotes a dose deposited in a tissue from radiation coming from the radionuclides contained in another tissue. The relative values of the two dose components depend upon the organ, the emitted radiation type and its energy. The positron radiation emitted in positron emission tomography (PET) is absorbed in short distances from the emission point. Thus, the self-absorption component is the dominant dose component for source regions. A numerical example of this is given in the Results. Organ selfabsorption is dominated by organ mass, as demonstrated in Pamphlet 11 of the Committee on Medical Internal Radiation Dose (MIRD) [27], which provides a guidance on how patient-specific scaling of reference radionuclide SAF values is to be done. This scaling requires only the mass of the target (=source) region and is, hence, otherwise independent of the organ topology of an individual. The cross-fire (except the cross-fire from blood and total body), on the contrary, is influenced by the organ topology. Thus, this study focuses on the anatomy-dependent cross-fire components of organ absorbed doses. We evaluate whether the accuracy of internal dose estimations can be efficiently improved with less effort than would be required were we to segment a patientspecific model.

Whalen et al. [28] demonstrated the feasibility of reducing the uncertainties in organ volumes to $21-29 \%$ for all organs, except spleen, by using the trunk height as a parameter to match a patient with a library of computational phantoms. Whalen et al. [28] also showed that the uncertainties in organ volumes can be further reduced, if ventral cavity volumes are matched. Little work has been done to investigate the possible reduction in the uncertainty of estimated dose that is achievable by adjusting reference phantoms to match the individual patient.

The objective of this work is to check the feasibility of reducing the uncertainties in the cross-fire component of organ internal doses by adjusting a reference phantom to selected external dimensions of individuals. Since it is beyond the scope of this study to evaluate the differences of the cross-fire SAFs for all potential source/target region combinations, as an example, we focused on the biokinetic behaviour of a novel radiopharmaceutical (S)-4$\left(3-{ }^{18} \mathrm{~F}\right.$-fluoropropyl)-L-glutamic acid $\left({ }^{18} \mathrm{~F}-\mathrm{FSPG}\right)$, for which comprehensive datasets were available.

\section{Methods and materials}

\subsection{Voxel and polygon-surface based ICRP adult male reference computational phantoms}

ICRP Publication 110 [1] gives the detailed description of the voxel ICRP adult reference computational phantoms, their applications and possible limitations. These models are the official computational models of reference adults, adopted by ICRP [1]. Voxelbased computational phantoms are limited in their flexibility though $[2,3]$. Such phantoms can be modified only by increasing or decreasing the dimensions of each voxel [29] or adding or removing of voxel layers in an organ. Selective adjustment or scaling of various body parts of a voxel phantom by different factors cannot be easily done. Thus, as a base model in the current work we used the polygon-surface ICRP male reference phantom [30]. It was developed by converting the voxel ICRP male reference phantom to a polygon-surface format. As a BREP model, the polygon-surface phantom can be relatively easily modified and deformed $[2,3]$. Note that the polygon-surface ICRP male reference phantom used here and described by Yeom et al. [30] is not the final version, since it is currently under development [31,32]. Analogous to the abbreviation for the voxel ICRP adult male reference computational phantom used by e.g. Schlattl et al. [33] and utilised in this work (RCP-AM), we denote the polygon-surface ICRP adult male reference computational phantom as P-RCP-AM in the following.

\subsection{Target patient-specific voxel models and adjusted characteristics}

Two voxel models from the Helmholtz Zentrum München (HMGU) model library were considered to be fictitious individual target patients in this work. We refer to these models as to patient-specific phantoms. The proposed approach was to apply relatively simple adjustments to P-RCP-AM and, thus, to make PRCP-AM more specific to the two selected individual target patients. The goodness of this approach was subsequently evaluated depending on how close the organ doses for the adjusted reference phantoms are to the real patient-specific doses. To test the approach on phantoms segmented from individuals with potentially high differences in anatomy, the biggest available model Visible Human [6] and the smallest available model Irene [5,6] (see Fig. 1) were selected as fictitious patients. The BMI of Visible Human and Irene is equal to $31.9 \mathrm{~kg} / \mathrm{m}^{2}$ and $19.2 \mathrm{~kg} / \mathrm{m}^{2}$, respectively. Despite P-RCP-AM being male, the female model Irene was chosen since no male model with similarly small BMI is available in the HMGU model library. Thus the doses for gender-specific organs could not be compared between Irene and P-RCP-AM adjusted to Irene. The comparison of the doses for other target regions appears to be appropriate though.

The Visible Human model used here was segmented from the CT images obtained from the National Library of Medicine's Visible Human Project. This phantom is a partial-body phantom covering 


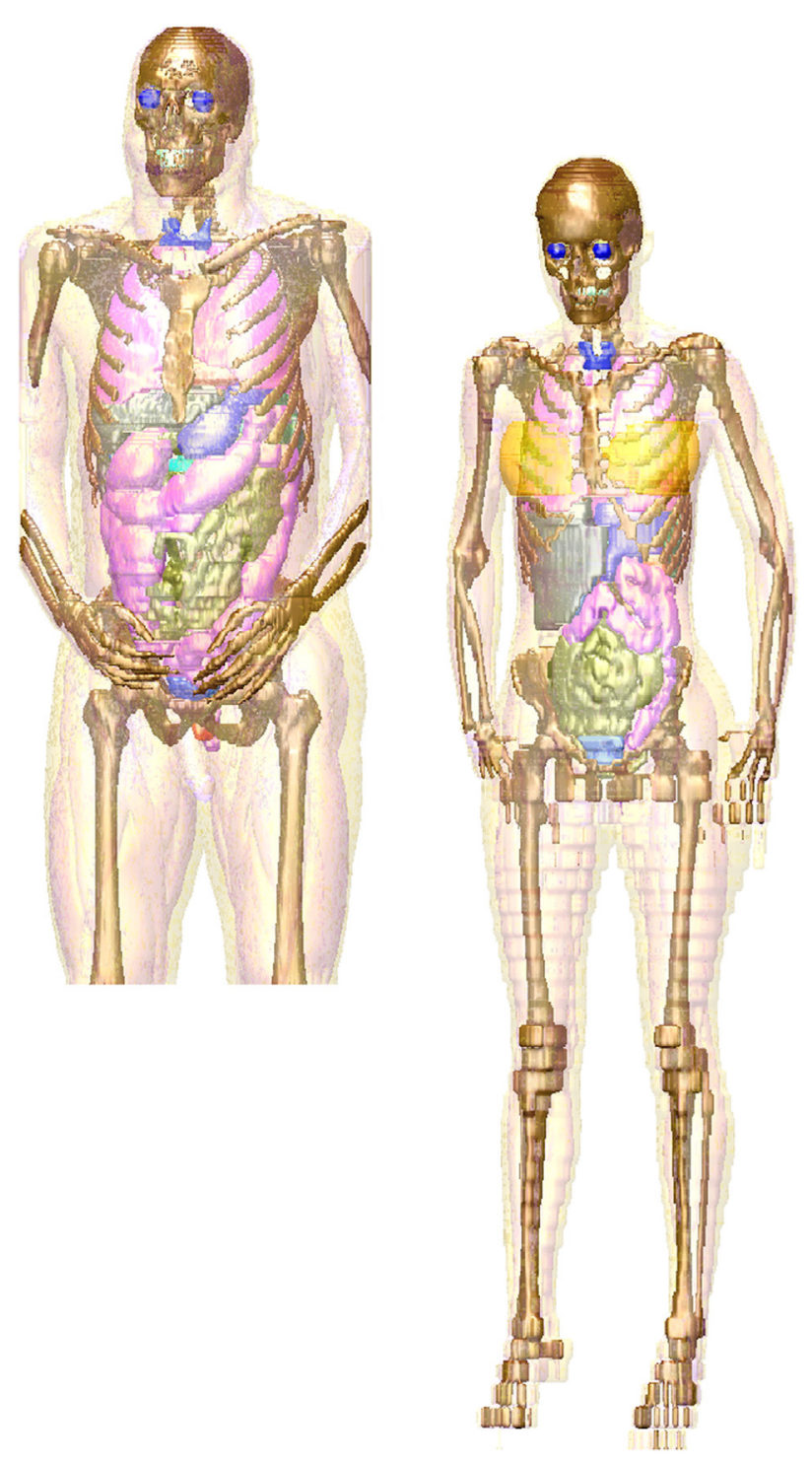

Fig. 1. Voxel phantoms Visible Human (left) and Irene (right) [33], denoted here as Pat1 and Pat2, respectively.

the head to mid-thigh. It is described in detail by Zankl et al. [6]. The Visible Human phantom has been constructed from an individual who had only one testicle. This circumstance resulted in a testes mass that is lower than the reference value (25.5 g compared to $35 \mathrm{~g}$ ). However, for the radiopharmaceutical under consideration the testes are not a source region. Hence, the testes dose is only due to cross-fire irradiation. Consequently, this lower target region mass does not have an impact. Another voxel phantom of the same individual described by Xu et al. [34] exists, segmented from whole body colour photographs. Irene has been constructed from the CT images of a patient, covering the whole body $[5,6]$.

For the sake of simplicity and to emphasize that Visible Human and Irene represent fictitious individual patients in this work, we denote them as Pat1 and Pat2, respectively. The following dimensions of P-RCP-AM [30] were adjusted to the corresponding individual characteristics of Pat1 and Pat2: maximum distance between the iliac crest and the clavicles, total height, front-toback distance and width of the rib cage (both are the corresponding maximum dimensions on the level of the xiphoid process). Sitting height or trunk height along with total standing height were previously considered by various authors to create a family of human computational phantoms or to match a patient with a library of phantoms $[8,10-12,15,17,18,28]$. The distance between the iliac crest and the clavicles was selected as an analogue to the trunk height. Whalen et al. [28] matched ventral cavity volumes. To scale this body part, we used the front-to-back distance and width of the rib cage, since these dimensions are relatively easy to measure. The dimensions of the rib cage correspond to those of the skeleton, not the external body dimensions. It is expected that the dimensions of the volume spanned by the rib cage correlate with the relative locations of the organs inside the rib cage better than those of the external body, especially for overweight individuals with a thick layer of subcutaneous fat. The front-to-back distance and the width of the rib cage (skeleton) can be estimated by subtraction of the approximate thickness of the subcutaneous fat from the corresponding external body dimensions. Alternatively, the rib cage dimensions can be determined easily from e.g. a CT scan which usually accompanies the actual PET diagnosis. The two new phantoms, obtained by matching PRCP-AM to Pat1 and Pat2, are indicated as Pat1M and Pat2M, respectively. Table 1 summarises all abbreviations used for naming the phantoms in this work. The selected set of adjustments was restricted to individual characteristics that are relatively easy to measure in actual patients. The head of the phantoms was not scaled in any dimension. The expected variability in the size of a head should not be high. In this study, as in a test study, only the dimensions with high expected variability are considered. Since the female individual Pat2 is represented by the male model Pat2M with only limited set of adjustments made, waist to hip ratio is not matched, bone size and muscle distribution are different. Additionally, gender-specific organs as uterus, gonads or prostate are excluded from the analysis.

All adjustments were made in the 3D modelling software Rhinoceros ${ }^{\circledR} 4.0$ (McNeel North America, Seattle, WA), which provides powerful tools for creating and editing non-uniform rational Bspline curves, surfaces and polygon meshes. The adjustments were applied in three steps, which are schematically shown in Fig. 2. At the first step the distance between the iliac crest and the clavicles and the total height were matched. This was achieved by scaling the rib cage, the spine, the clavicles, the sternum, the scapulae and all the organs inside the rib cage on the level of thoracic and lumbar spine, including the small intestine and the colon, in superior-inferior directions by a factor needed to match the distance between the iliac crest and the clavicles. For convenience, prior to the first scaling step, the pelvis along with the legs, the bladder, the prostate, the testes and the inguinal lymphatic nodes were moved in inferior direction, as shown in Fig. 2. After matching the distance between the iliac crest and the clavicles the phantom was re-unified. If needed, the first scaling step was followed by a scaling of the legs in inferior direction to match the total height. At the second step all the aforementioned organs, except the arms,

Table 1

Abbreviations used for indicating the computational models in this work.

\begin{tabular}{|c|c|}
\hline Description & $\begin{array}{l}\text { Used } \\
\text { abbreviation }\end{array}$ \\
\hline Voxel ICRP adult male reference computational phantom [1] & RCP-AM \\
\hline $\begin{array}{l}\text { Polygon-surface ICRP adult male reference computational } \\
\text { phantom [30] }\end{array}$ & P-RCP-AM \\
\hline Visible Human [6] (fictitious patient 1 ) & Pat1 \\
\hline Irene $[5,6]$ (fictitious patient 2 ) & Pat2 \\
\hline $\begin{array}{l}\text { Phantom obtained via adjustments of polygon-surface ICRP } \\
\text { adult male reference computational phantom to fit selected } \\
\text { characteristics of Visible Human }\end{array}$ & Pat1M \\
\hline $\begin{array}{l}\text { Phantom obtained via adjustments of polygon-surface ICRP } \\
\text { adult male reference computational phantom to fit selected } \\
\text { characteristics of Irene }\end{array}$ & Pat2M \\
\hline
\end{tabular}



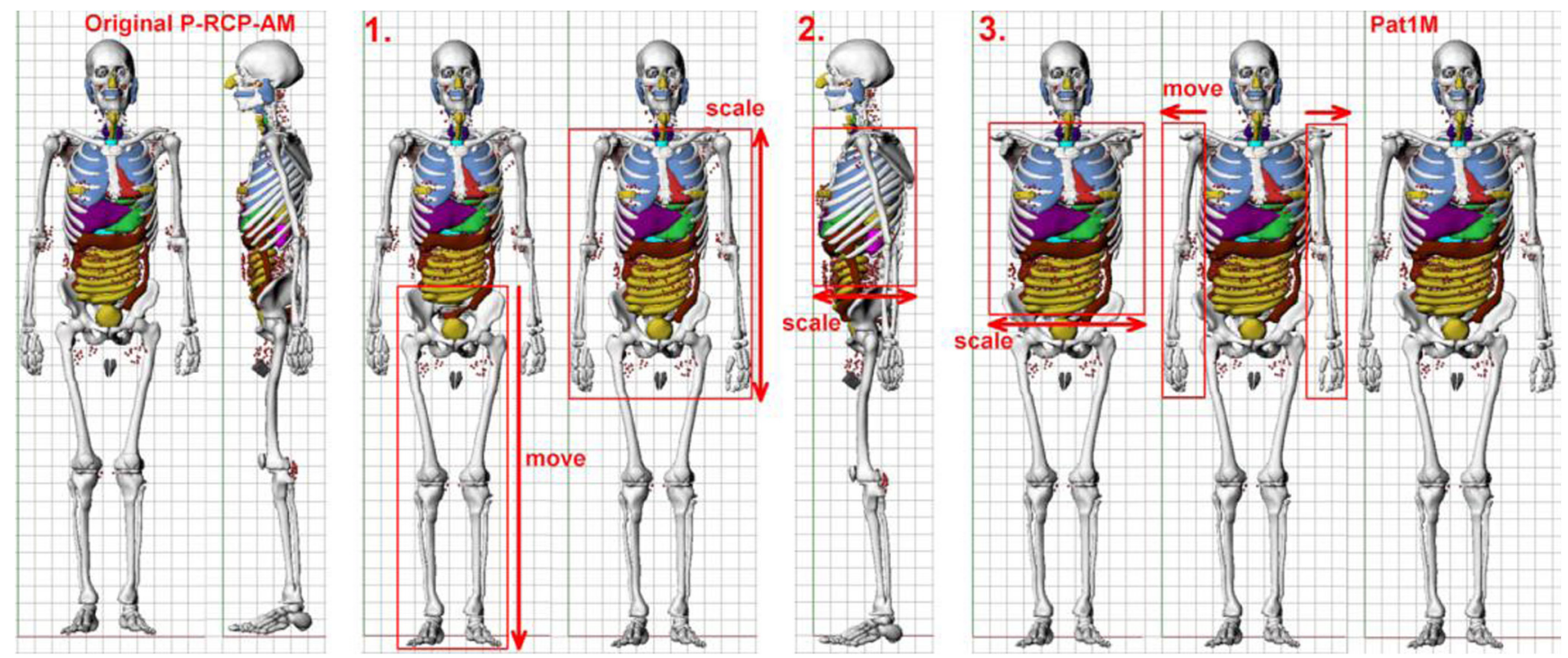

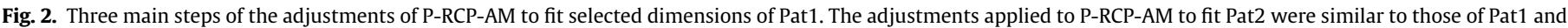
thus are not shown here.

were scaled together with the rib cage in anterior-posterior direction to fit the outer front-to-back distance of the rib cages of Pat1 and Pat2. At the third step the scaling in lateral dimension was applied to match the outer width of the rib cages of the two patients considered. The arms were not scaled, but moved laterally to fit the scaled thorax. The scaling was done with the Scale 1D command. Organ intersections were then found with the MeshIntersect command (Rhinoceros ${ }^{\circledR}$ 4.0) and eliminated by applying some minor adjustments of the intersecting organs with the Smooth command and/or Mesh Edit and Mesh Repair Tools of Rhinoceros ${ }^{\circledR}$ 4.0. To scale and repair the skin mesh, the Scale 1D command as well as the Mesh Edit and Mesh Repair Tools along with the Smooth command were employed. No further adjustments of individual organ positions and sizes of Pat1M and Pat2M to match the corresponding values of Pat 1 and Pat2 were applied.

\subsection{Monte Carlo calculations}

The Monte Carlo code EGSnrc [35] with a user code developed at the HMGU was employed in this work. The SAFs for homogeneous volume sources of monoenergetic photons and electrons were calculated in the geometry of the six computational phantoms. The EGSnrc system has been previously tested against other Monte Carlo codes [1,36-38]. The user code has been previously utilised and benchmarked in various works to compute dose conversion coefficients for public, occupational and medical exposures. The detailed description of the user code can be found elsewhere [7,26,33]. A total of 18 electron energies, ranging from $10 \mathrm{keV}$ to $1 \mathrm{MeV}$, and one photon energy of $511 \mathrm{keV}$ were considered. The simulated energies were selected to cover the beta spectra of ${ }^{11} \mathrm{C}$ - and ${ }^{18} \mathrm{~F}$-labeled compounds, which are commonly used in PET, and the energy of annihilation photons. The electron energies were in accordance to ICRP Publication 133 [26] with an additional energy of $250 \mathrm{keV}$. For photon internal sources and electron internal sources in the energy range of $10-600 \mathrm{keV}, 40$ million histories were simulated per source region and energy. For electron energies of $800 \mathrm{keV}$ and higher the number of simulated histories was reduced to 10 million for the sake of computational time. Photon histories were terminated at $2 \mathrm{keV}$. Electron histories with kinetic energies less than $50 \mathrm{keV}$ were followed down to $2 \mathrm{keV}$. All other electron histories were terminated at $20 \mathrm{keV}$. The source regions considered included total blood, heart wall, heart contents, kidneys, liver, pancreas, salivary glands, spleen, stomach wall, total body, thyroid and urinary bladder content. The source region "total blood" comprised blood vessels, if present in the phantom, and regional blood volumes (RBVs) of organs and tissues. Target regions included all organs and tissues segmented in the phantoms.

Since the EGSnrc user code utilises voxel geometry, all polygonsurface phantoms considered had to be voxelised. Using the software Binvox (Patrick Min, http://www.patrickmin.com/binvox/) [39] all polygon-surface organs have been transferred into individual organ voxel models with $1 \times 1 \times 1 \mathrm{~mm}^{3}$ voxel size. They were then combined to complete phantoms using an in-house IDL-based (IDL ${ }^{\circledR}$, Exelis Visual Information Solutions, Inc., version 8.2.1) program. For simplicity, here and in the following we use the same abbreviations for the polygon-surface models and their voxelised versions, since only the latter were used for the Monte Carlo calculations. Media elemental compositions and densities from ICRP Publication 110 [1] were used in P-RCP-AM, Pat1M and Pat2M. For each target phantom, Pat1 and Pat2, three models approximating it were considered: RCP-AM, P-RCP-AM and Pat1M for Pat1, RCP-AM, P-RCP-AM and Pat2M for Pat2 (see Table 1). Table 2 summarises selected properties of the employed phantoms.

It was previously shown that blood activity has a substantial effect on absorbed doses for highly vascular organs, which are not source regions [40]. As mentioned above, total blood as source region consists of blood vessels and RBVs. The total blood in Pat1 and Pat 2 originally did not comprise RBVs. To include RBVs into the total blood, mass fractions of blood in different organs and tissues are required. Nadler's formula [41] was applied to calculate the total blood volumes for Pat 1 and Pat2. Total blood masses were computed with the blood density from ICRU Report 46 [42] and ICRP Publication 110 [1] $\left(1.06 \mathrm{~g} / \mathrm{cm}^{3}\right)$. Using the resulting total blood masses, reference RBVs from ICRP Publication 89 [43] and the organ masses of Pat 1 and Pat2, mass fractions of blood were calculated for organs and tissues of the aforementioned phantoms. These fractions were subsequently considered in the Monte Carlo calculations.

\subsubsection{Mass-correction of SAFs derived for whole-body anatomical models}

The MIRD formalism [44] assumes reference mass values for the organs (source and target) and the total body. MIRD Pamphlet 11 
Table 2

Selected properties of considered computational phantoms.

\begin{tabular}{|c|c|c|c|c|}
\hline Phantom & Height, $[\mathrm{cm}]$ & Weight, [kg] & Number of segmented regions & Voxel dimensions, $\left[\mathrm{mm}^{3}\right]$ \\
\hline Pat1 & $180.0(125.0)^{\mathrm{a}}$ & $103.2(89.1)^{\mathrm{a}}$ & 131 & $0.9 \times 0.9 \times 5.0$ \\
\hline Pat2 & 163.0 & 51.1 & 62 & $1.9 \times 1.9 \times 5.0$ \\
\hline RCP-AM & 176.0 & 73.0 & 138 & $2.1 \times 2.1 \times 8.0$ \\
\hline P-RCP-AM & 176.4 & 73.6 & 111 & $1.0 \times 1.0 \times 1.0$ \\
\hline Pat1M & 180.2 & 95.6 & 111 & $1.0 \times 1.0 \times 1.0$ \\
\hline Pat2M & 162.4 & 51.1 & 111 & $1.0 \times 1.0 \times 1.0$ \\
\hline
\end{tabular}

a Pat1 is a partial-body phantom. The values in parentheses correspond to the available part of the phantom, without parentheses to the actual height and weight of the individual.

[27] provides guidance on how patient-specific scaling of reference radionuclide $S$ values (and eventually SAF values) is to be done for the electron and photon component of the emission spectrum. Based on this guidance and results of extensive analysis of the mass dependences of photon [24] and electron [45] SAFs, the SAFs derived for RCP-AM, P-RCP-AM, Pat1M and Pat2M were masscorrected. This was done to transfer the simulated values to the investigated fictitious patients (Pat1 and Pat2), who have organ masses differing from those of RCP-AM, P-RCP-AM, Pat1M and Pat2M. The mass-dependences of the SAFs for whole-body phantoms, demonstrated by Petoussi-Henss et al. [24] and Zankl et al. [45], include self-irradiation, i.e. source equal to target for both photons and electrons and the case of electron total-body source with the target being a part of it.

If the source is equal to the target, the SAFs for electrons are inversely proportional to the mass of the target (=source) and the SAFs for photons are proportional to the (mass) $)^{-2 / 3}$ of the target (=source). The corresponding mass-corrections of SAFs were applied in this case to account for the differences in the masses of target $=$ source between Pat 1 and Pat 2 and the models approximating them.

Before the SAFs for electrons were explicitly derived from particle transport calculations, an approximation given by ICRP [46] was commonly used. According to it, the SAFs for all internal organs as targets and a total body source are approximately equal to the inverse of the total body mass [46]. The validity of this approximation has also been demonstrated by Zankl et al. [45]. The SAFs derived for a total body source of electrons and internal organs as targets were corrected in this way to account for differences in total body masses of RCP-AM, P-RCP-AM, Pat1M (Pat2M) relative to Pat1 (Pat2).

\subsubsection{Correction of SAFs evaluated with a partial-body anatomical model}

As mentioned above, the Visible Human phantom (Pat1) is a partial-body phantom, from head to mid-thigh. Thus the SAFs simulated for this model had to be adjusted to correspond to a wholebody model. Several cases had to be considered. If the source and the target are both entirely inside the partial-body model, the SAFs of the whole-body model would not differ from those of the partial-body model. Hence, the same mass-correction as for the whole-body models was applied in this case. The second case is for total body as a source and a target being entirely inside the partial-body model. As mentioned above, the electron SAFs in this case are approximately equal to the inverse of the total body mass $[45,46]$. Hence, the SAFs evaluated with a phantom comprising of only a portion of the body are overestimated and should be corrected, such that they correspond to the inverse mass of the total body of the whole person, rather than to the mass of the phantom only. The same is applied here for photons. If the source is another extended region ( $\neq$ total body) that is partly outside the partialbody phantom, e.g. total blood or muscles, and the target is entirely inside the phantom, the SAFs are inversely proportional to the total mass of the source region. Thus, the SAFs simulated for partialbody phantoms should be corrected in this case to account for the missing mass of the extended source region. If the source is entirely inside the phantom and the target is an extended region that is only partly inside the available part of the phantom, e.g. total body, skin and muscles, the reciprocity principle, introduced by Cristy and Eckerman [47] for photons and confirmed for electrons by Zankl et al. [45], suggests that the situation is the same as when the target is entirely inside the partial-body phantom and the source is not. Hence, the SAFs are inversely proportional to the total mass of the target and the SAFs evaluated for partialbody phantoms should be corrected for the missing target mass. If both source and target extend beyond the phantom, electron and photon SAFs are inversely proportional to the highest mass (source or target). Thus, the SAFs simulated for a partial-body phantom had to be corrected for the missing mass of a heavier region (source or target). All the aforementioned cases of masscorrection of the SAFs were implemented in the developed inhouse program for the internal organ absorbed-dose calculations and applied in this work.

\subsection{Activity data and internal organ dose calculation}

For internal dosimetry in this work we employed five sets of time-integrated activity coefficients derived in a previous study [40] for a novel radiopharmaceutical for PET/CT imaging ${ }^{18} \mathrm{~F}$ FSPG. The case of blood being a distinct source region was considered [40].

We calculated absorbed-dose coefficients five times (i.e. once for each set of activity data) for each of the models Pat1, RCPAM, P-RCP-AM and Pat1M with the SAFs corrected to correspond to the masses of Pat1 (see Sections 2.3.1 and 2.3.2). For each set of activity data the dose differences between RCP-AM, P-RCP-AM and Pat1M relative to Pat1 were computed. Average, maximum and minimum values of these differences are reported. The same procedure was done for the models Pat2, RCP-AM, P-RCP-AM and Pat2M with SAFs scaled to the masses of Pat2. The study [40] was the source of the activity data only. No attempt was made to consider the individual anatomy of the healthy volunteers from the previous study [40] or to track their doses.

An in-house MATLAB-based (MATLAB, version 8.0.0.783 (R2012b), The MathWorks, Inc., Natick Massachusetts, 2012) program was used to calculate organ absorbed-dose coefficients, according to the MIRD system [44]. ${ }^{18} \mathrm{~F}$ is a $\beta^{+}$emitter $\left(E_{\text {mean }}=249.8 \mathrm{keV}, E_{\max }=633.5 \mathrm{keV}\right)$. The detailed beta spectrum of ${ }^{18} \mathrm{~F}$ from ICRP Publication 107 [48] and a single photon energy at $511 \mathrm{keV}$ (annihilation photons) were utilised for the dosimetric calculations. The SAFs corresponding to those intermediate electron energies which were not explicitly considered in Monte Carlo calculations were derived via a logarithmic interpolation of the simulated data. The curves describing the dependence of the explicitly calculated SAFs upon the electron energy are smooth and, hence, the interpolation was appropriate. The SAFs for bladder 
contents as source and bladder wall as target depend upon the volume of the bladder contents and the thickness of the bladder wall $[49,50]$. The SAFs simulated for the computational phantoms in this work do not reflect these dependences, though, and correspond to fixed volumes of the bladder contents, which amount to 39.3 and $24.3 \mathrm{~cm}^{3}$ for Pat 1 and Pat2, respectively. Since these volumes are substantially smaller than the reference volume of the bladder contents $\left(192.3 \mathrm{~cm}^{3}\right)$ [1] and no scaling was done to the bladder, we did not use the SAF values obtained from the phantoms, but exploited those calculated by Andersson et al. [51].

In Pat1 breast was not segmented. Hence for breast in Pat 1 the total breast of the RCP-AM was used. The heart wall of Pat1 and Pat2 was surrogated by heart (wall and content). Pat2 is a computational phantom representing a female individual whereas P-RCP$\mathrm{AM}$ and, consequently, Pat2M represent male individuals. Thus, the doses for gonads, uterus or prostate cannot be compared. Because P-RCP-AM is still under development [30-32], some complex structures, e.g. muscles, are not present in P-RCP-AM, Pat1M and Pat2M. Thus subcutaneous fat and skeletal muscle were considered together as only one tissue (residual tissue). The skeletal muscle in P-RCP-AM, Pat1M and Pat2M was surrogated by residual tissue in the dose computation. The elemental composition and the density of the residual tissue were adjusted to correspond to a mixture of muscles and adipose (66\% and 34\%, respectively, for the parenchyma mass fractions).

\section{Results}

\subsection{Anatomical models Pat1M and Pat2M}

A representative subset of four slices of the voxelised P-RCP-AM is presented in Fig. 3. The grey values on the images correspond to the organ labels of the phantom P-RCP-AM. As can be seen from Fig. 3 , the contours of the organs of P-RCP-AM are smooth and the voxel size of $1 \times 1 \times 1 \mathrm{~mm}^{3}$ is sufficient for realistic representations of most of the organs.

Fig. 4 shows the polygon-surface model P-RCP-AM along with Pat1M and Pat2M that were obtained via the adjustment of $\mathrm{P}$ RCP-AM to some individual characteristics of Pat 1 and Pat2. The adjusted dimensions are summarised in Table 3 . It can be seen from Fig. 4 that the adjusted phantoms Pat1M and Pat2M retain their anatomic realism.

\subsection{Calculated organ absorbed-dose coefficients}

The statistical uncertainties of the Monte Carlo calculations performed notably depend on the absolute values of the absorbed fractions (AF). For the target regions located distantly from the source only a small fraction of energy released from the source can reach the target. This results in low AF and higher statistical uncertainties. For the calculations done here, the coefficients of variance for $\mathrm{AF} \geq 10^{-3}$ were below $0.5 \%$, in most cases even below $0.25 \%$. For the values $10^{-4} \leq \mathrm{AF}<10^{-3}$, the coefficients of variance were below $1.5 \%$, for $10^{-5} \leq \mathrm{AF}<10^{-4}$ the coefficients of variance amount to $2.5 \%$ at most, and for $10^{-6} \leq \mathrm{AF}<10^{-5}$ they were below $10 \%$.

The five sets of time-integrated activity coefficients derived in [40] and the simulated SAFs for the six computational phantoms were used for dose computation. We assessed absorbed-dose coefficients 40 times in total: five times for each of the models Pat1, RCP-AM, P-RCP-AM and Pat1M with SAFs scaled to the masses of Pat1 and five times for each of the models Pat2, RCP-AM, P-RCPAM and Pat2M with SAFs scaled to the masses of Pat2.

To distinguish the impact of the individual organ topology on the computed organ absorbed-dose coefficients, the selfabsorption and the cross-fire components of the respective organ absorbed-dose coefficients are given separately in the following.

For each set of activity data the dose differences of RCP-AM, PRCP-AM and Pat $1 \mathrm{M}$ relative to Pat 1 and of RCP-AM, P-RCP-AM and Pat2M relative to Pat2 were computed. We calculated average, minimum and maximum values of these relative differences for the cross-fire components of the organ dose coefficients. They are summarised in Table 4 and Table 5.

\subsubsection{Self-absorption component of absorbed-dose coefficients}

The source regions fixating the radiopharmaceutical ${ }^{18} \mathrm{~F}-\mathrm{FSPG}$ are: stomach wall, liver, thyroid, salivary glands, heart wall, kidneys, urinary bladder-contents, pancreas, spleen and blood. Due to the accumulation of activity in these regions and relatively low energies of the emitted positrons in case of ${ }^{18} \mathrm{~F}\left(E_{\max }\left(\beta^{+}\right)\right.$ $=633.5 \mathrm{keV}$ ), the doses received by the source regions are predominantly due to self-absorption. The calculated absorbed-dose coefficients due to self-absorption along with the relative deviation from the respective values calculated for Pat 1 and Pat 2 and the absolute contributions of self-absorption to the total organ absorbed-dose coefficients are presented in Fig. 5. Note that the absorbed-dose coefficients from Fig. 5 are computed with one set of activity data. The corresponding figures obtained using the other four sets of activity data are similar and, hence, are not presented here.

Except for heart wall and stomach wall, no substantial differences in the self-absorption dose coefficients are observed. The contributions of self-absorption to total absorbed-dose coefficients

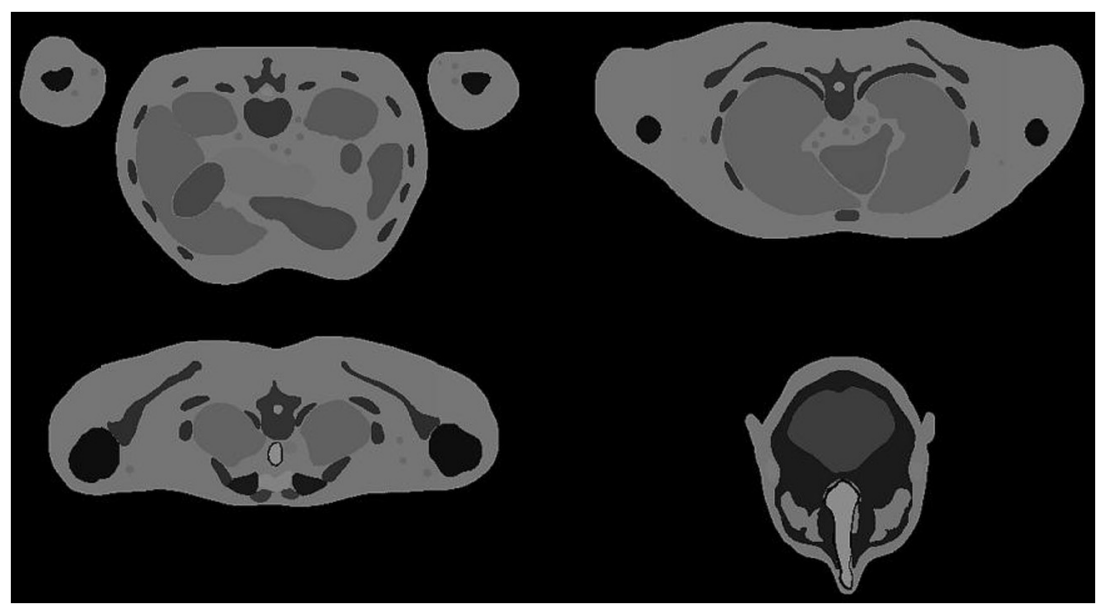

Fig. 3. A subset of four slices of P-RCP-AM. The voxel size is $1 \times 1 \times 1 \mathrm{~mm}^{3}$. Grey values correspond to the organ labels. 


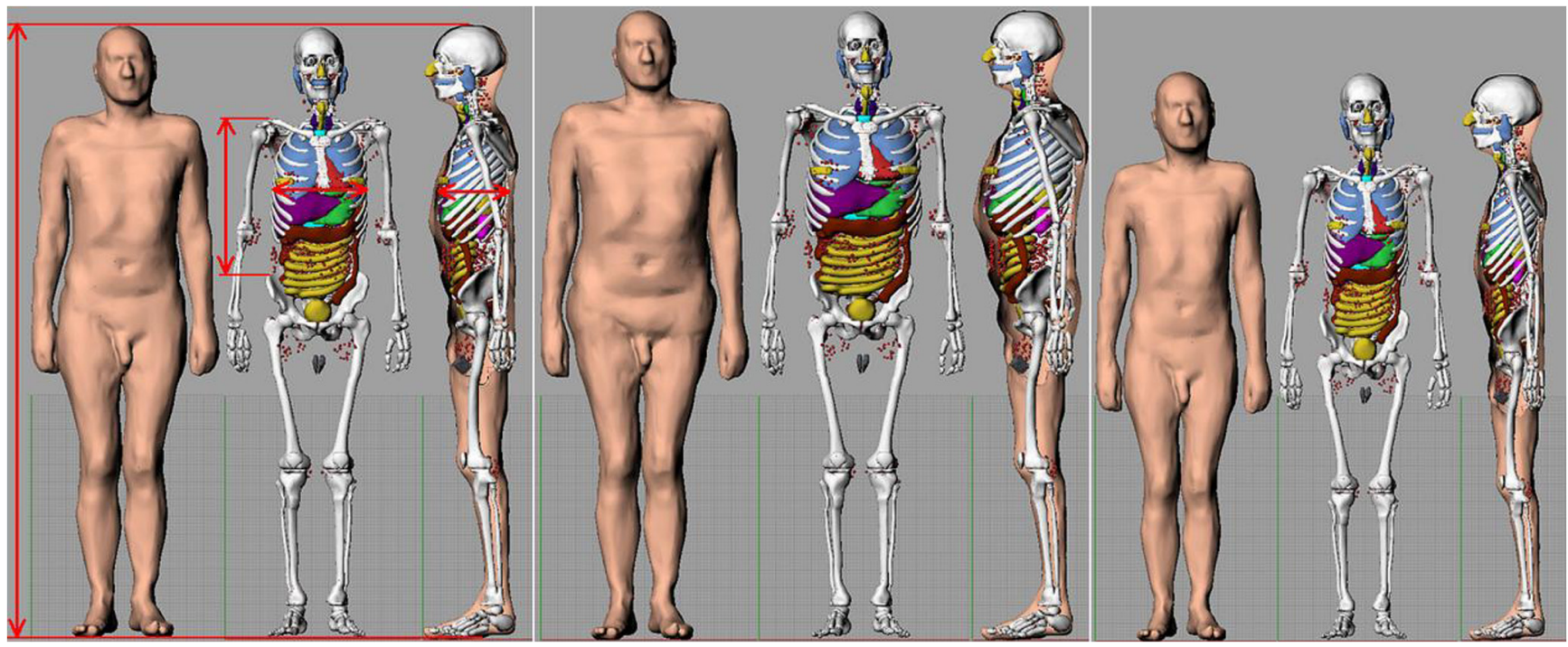

Fig. 4. P-RCP-AM with the selected dimensions and the result of its adjustment to Pat 1 and Pat2 (from left to right).

Table 3

Selected dimensions of Pat1 and Pat2, [cm] and the corresponding matched dimensions of Pat1M and Pat2M, [cm].

\begin{tabular}{|c|c|c|c|c|c|c|}
\hline Adjusted characteristic & Pat1 & Pat1M & Difference, \% & Pat2 & Pat2M & Difference, \% \\
\hline Distance between the iliac crest and clavicles & 46.0 & 46.2 & $0.4 \%$ & 40.0 & 39.7 & $-0.8 \%$ \\
\hline Front-to-back distance of the rib cage & 23.6 & 23.7 & $0.3 \%$ & 15.0 & 15.1 & $0.5 \%$ \\
\hline Width of the rib cage & 32.7 & 32.7 & $0.1 \%$ & 23.6 & 23.6 & $0.2 \%$ \\
\hline Height & 180.0 & 180.2 & $0.1 \%$ & 163.0 & 162.4 & $-0.4 \%$ \\
\hline
\end{tabular}

The differences are given in \% of the original dimensions of Pat 1 and Pat2.

are shown in Fig. 5. Notably, the contribution is particularly high for kidneys and pancreas.

\subsubsection{Cross-fire component of absorbed-dose coefficients}

The subset of the calculated absorbed-dose coefficients from cross-fire along with the relative deviations from those calculated for Pat1 and Pat2 employing one set of activity data are presented in Fig. 6 and Fig. 7, respectively. The corresponding figures for the other four sets of activity data are similar to those of Fig. 6 and Fig. 7 and, therefore, are not presented here. The same set of activity data is used for Fig. 5, Fig. 6 and Fig. 7.

Table 4 and Table 5 show the relative differences in the calculated absorbed-dose coefficients from cross-fire for Pat1 and Pat2, respectively, and the models approximating Pat1 and Pat2. The values given in Table 4 and Table 5 are computed as average, minimum and maximum within dose differences calculated with five sets of activity data. For the majority of organs of Pat1M (Table 4) and Pat2M (Table 5) the relative deviations in cross-fire absorbed-dose coefficients from those calculated for Pat1 and Pat2 are lower than the respective dose differences between the target phantoms Pat1 and Pat2 and the reference phantoms (RCP-AM, P-RCP-AM).

The computed relative differences are similar for all activity datasets. No differences in absorbed-dose coefficients for urinary bladder wall were observed, since the same SAFs for bladder contents as source and bladder wall as target derived by Andersson et al. [51] were employed. The breast of RCP-AM was used as a surrogate for the breast of Pat1. Hence, no significant differences exist between Pat1 and RCP-AM, while the differences between Pat1 and Pat $1 \mathrm{M}$ are below $18.1 \%$. For Pat 1 the relative differences in crossfire absorbed-dose coefficients of liver, extrathoracic airways, kidneys, pancreas and eyes decreased from about 30.0-40.0\% for the reference phantoms RCP-AM and P-RCP-AM to less than $10.0 \%$ for the matched phantom Pat1M. For oesophagus, brain, adrenals, heart wall, spleen, thymus and spinal cord the cross-fire dose differences between Pat1 and the reference phantoms amount to approximately $40.0 \%$, while the corresponding differences between Pat1 and Pat1M are below $15.0 \%$. For Pat1 the differences in absorbed-dose coefficients of gall bladder wall amount to $80.6 \%$ and $75.5 \%$ on average for RCP-AM and P-RCP-AM, respectively. The corresponding differences relative to Pat1M are below $30.0 \%$ on average. For active bone marrow, thyroid and salivary glands the calculated relative dose differences between Pat1M and Pat1 are only half of those between reference phantoms and Pat1. For lungs the computed dose differences decreased on average from 21-24\% between Pat1 and reference phantoms to $-3 \%$ between Pat1 and Pat1M. The differences in the calculated cross-fire absorbed-dose coefficients for stomach wall and small intestine wall amount, respectively, to $12.4 \%$ and $14.3 \%$ on average between Pat 1 and Pat1M. The corresponding differences between Pat1 and both reference phantoms are within $54.7 \%$ and $57.9 \%$ on average. We observed better agreement between Pat 1 and the reference phantoms (RCP-AM and P-RCP-AM) in the absorbed-dose coefficients for gonads, prostate, skeleton and colon wall. Absorbeddose coefficients for all organs, except gonads and gall bladder wall are consistent between Pat1 and Pat1M within 25.0\% (Table 4).

For Pat2 the differences in the cross-fire absorbed-dose coefficients for kidneys, pancreas, brain, small intestine, thymus, and eye lenses relative to Pat2M are approximately lower by a factor of two compared to those relative to the reference phantoms. As a general finding for all sets of activity data, the differences in the absorbed-dose coefficients for lungs, stomach wall (crossfire), adrenals, extrathoracic airways (ET) and spinal cord decreased from approximately $30.0 \%$ between RCP-AM, P-RCP$\mathrm{AM}$ and Pat2 to less than $10.0 \%$ between Pat2M and Pat2. The absorbed-dose coefficients for eyes and muscles in Pat2M are con- 
Table 4

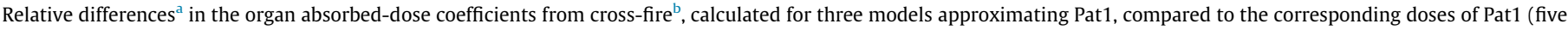
sets of activity data were used).

\begin{tabular}{|c|c|c|c|c|c|c|c|c|c|}
\hline & \multicolumn{3}{|l|}{ RCP-AM } & \multicolumn{3}{|c|}{ P-RCP-AM } & \multicolumn{3}{|l|}{ Pat1M } \\
\hline & Mean & Min & Max & Mean & Min & Max & Mean & Min & Max \\
\hline Active bone marrow & $38.1 \%$ & $32.7 \%$ & $46.3 \%$ & $35.2 \%$ & $30.2 \%$ & $43.3 \%$ & $14.5 \%$ & $11.4 \%$ & $19.2 \%$ \\
\hline Colon wall & $8.6 \%$ & $5.8 \%$ & $10.3 \%$ & $11.0 \%$ & $7.2 \%$ & $13.1 \%$ & $-17.2 \%$ & $-18.4 \%$ & $-16.4 \%$ \\
\hline Lungs & $20.8 \%$ & $20.1 \%$ & $21.6 \%$ & $23.7 \%$ & $22.2 \%$ & $25.3 \%$ & $-3.1 \%$ & $-4.6 \%$ & $-1.0 \%$ \\
\hline Stomach wall ${ }^{\mathrm{C}}$ & $51.4 \%$ & $49.6 \%$ & $54.3 \%$ & $54.7 \%$ & $52.3 \%$ & $58.2 \%$ & $12.4 \%$ & $9.4 \%$ & $14.5 \%$ \\
\hline Breast, total & $0.0 \%$ & $0.0 \%$ & $0.0 \%$ & $4.5 \%$ & $4.0 \%$ & $5.5 \%$ & $-16.4 \%$ & $-18.1 \%$ & $-14.1 \%$ \\
\hline Gonads & $-20.6 \%$ & $-26.5 \%$ & $-18.3 \%$ & $-20.8 \%$ & $-26.3 \%$ & $-18.5 \%$ & $-28.4 \%$ & $-34.1 \%$ & $-26.2 \%$ \\
\hline Urinary bladder wall & $0.6 \%$ & $0.3 \%$ & $0.7 \%$ & $0.5 \%$ & $0.3 \%$ & $0.7 \%$ & $0.2 \%$ & $0.1 \%$ & $0.3 \%$ \\
\hline Oesophagus & $36.0 \%$ & $30.1 \%$ & $44.0 \%$ & $32.7 \%$ & $26.4 \%$ & $40.4 \%$ & $8.5 \%$ & $3.8 \%$ & $12.2 \%$ \\
\hline Liver $^{c}$ & $40.9 \%$ & $39.8 \%$ & $41.8 \%$ & $44.3 \%$ & $43.0 \%$ & $45.7 \%$ & $2.2 \%$ & $0.8 \%$ & $4.6 \%$ \\
\hline Thyroid $^{\mathrm{C}}$ & $50.7 \%$ & $46.8 \%$ & $57.1 \%$ & $51.5 \%$ & $47.3 \%$ & $58.5 \%$ & $18.9 \%$ & $16.8 \%$ & $21.4 \%$ \\
\hline Brain & $29.4 \%$ & $29.1 \%$ & $30.3 \%$ & $31.5 \%$ & $30.4 \%$ & $34.2 \%$ & $11.7 \%$ & $10.3 \%$ & $12.4 \%$ \\
\hline Salivary glands ${ }^{\mathrm{c}}$ & $15.7 \%$ & $15.5 \%$ & $16.1 \%$ & $20.7 \%$ & $19.8 \%$ & $22.5 \%$ & $-9.2 \%$ & $-10.6 \%$ & $-8.2 \%$ \\
\hline Skin, total & $12.7 \%$ & $9.5 \%$ & $14.6 \%$ & $11.9 \%$ & $9.1 \%$ & $13.3 \%$ & $-5.7 \%$ & $-10.9 \%$ & $-2.8 \%$ \\
\hline Adrenals & $38.7 \%$ & $31.5 \%$ & $46.9 \%$ & $40.1 \%$ & $32.7 \%$ & $48.4 \%$ & $3.3 \%$ & $-1.9 \%$ & $11.4 \%$ \\
\hline Extrathoracic airways (ET) & $19.7 \%$ & $19.2 \%$ & $20.7 \%$ & $25.1 \%$ & $24.0 \%$ & $27.9 \%$ & $3.2 \%$ & $0.9 \%$ & $4.5 \%$ \\
\hline Gall bladder wall & $80.6 \%$ & $67.8 \%$ & $88.7 \%$ & $75.5 \%$ & $64.4 \%$ & $83.7 \%$ & $29.4 \%$ & $23.2 \%$ & $35.6 \%$ \\
\hline Heart $^{\mathrm{c}}$ & $42.3 \%$ & $38.2 \%$ & $49.7 \%$ & $41.1 \%$ & $37.1 \%$ & $49.2 \%$ & $8.9 \%$ & $5.2 \%$ & $15.0 \%$ \\
\hline Kidneys $^{c}$ & $37.7 \%$ & $36.4 \%$ & $40.0 \%$ & $41.9 \%$ & $39.8 \%$ & $45.0 \%$ & $4.2 \%$ & $2.3 \%$ & $5.9 \%$ \\
\hline Pancreas $^{c}$ & $32.8 \%$ & $29.7 \%$ & $37.2 \%$ & $32.8 \%$ & $30.4 \%$ & $36.8 \%$ & $-6.5 \%$ & $-8.7 \%$ & $-2.9 \%$ \\
\hline Prostate & $-10.2 \%$ & $-11.1 \%$ & $-9.8 \%$ & $-12.8 \%$ & $-13.6 \%$ & $-12.4 \%$ & $-15.3 \%$ & $-16.2 \%$ & $-14.9 \%$ \\
\hline Small intestine wall & $57.9 \%$ & $47.7 \%$ & $65.8 \%$ & $45.3 \%$ & $34.4 \%$ & $54.2 \%$ & $14.0 \%$ & $5.5 \%$ & $22.9 \%$ \\
\hline Spleen $^{\mathrm{c}}$ & $39.9 \%$ & $30.9 \%$ & $47.3 \%$ & $43.2 \%$ & $33.0 \%$ & $51.7 \%$ & $6.6 \%$ & $-0.6 \%$ & $12.2 \%$ \\
\hline Thymus & $34.6 \%$ & $32.5 \%$ & $38.2 \%$ & $33.4 \%$ & $31.2 \%$ & $35.6 \%$ & $10.9 \%$ & $9.6 \%$ & $12.4 \%$ \\
\hline Spinal cord & $34.5 \%$ & $33.4 \%$ & $36.6 \%$ & $36.8 \%$ & $35.3 \%$ & $39.7 \%$ & $14.0 \%$ & $13.5 \%$ & $15.0 \%$ \\
\hline Skeleton, total & $-3.3 \%$ & $-4.4 \%$ & $-2.6 \%$ & $-3.6 \%$ & $-4.5 \%$ & $-3.0 \%$ & $-11.7 \%$ & $-13.3 \%$ & $-10.9 \%$ \\
\hline Muscle tissue & $23.1 \%$ & $22.4 \%$ & $24.8 \%$ & $31.7 \%$ & $29.0 \%$ & $35.4 \%$ & $18.5 \%$ & $16.8 \%$ & $20.6 \%$ \\
\hline Eyes & $18.7 \%$ & $15.9 \%$ & $20.1 \%$ & $27.2 \%$ & $25.0 \%$ & $28.5 \%$ & $4.9 \%$ & $-1.9 \%$ & $8.4 \%$ \\
\hline Eye lenses & $10.3 \%$ & $7.8 \%$ & $11.7 \%$ & $14.4 \%$ & $8.4 \%$ & $17.6 \%$ & $-9.9 \%$ & $-17.6 \%$ & $-5.9 \%$ \\
\hline
\end{tabular}

a The differences are expressed relative to the absorbed-dose coefficients from cross-fire for Pat1, since these are the patient-specific ones.

b Total absorbed-dose coefficients for source organs include in addition the contribution from the self-absorption, which is dominated by the organ mass and is otherwise

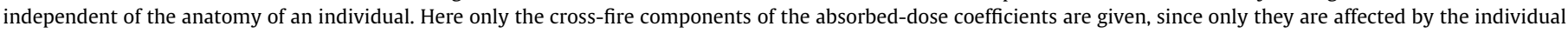
organ topology.

c These are the source organs for ${ }^{18} \mathrm{~F}-\mathrm{FSPG}$.

For $\mathrm{AF} \geq 10^{-4}$ the coefficients of variance were $1.5 \%$ at most, for $10^{-5} \leq \mathrm{AF}<10^{-4}$ the coefficients of variance were below $2.5 \%$. More information on statistical uncertainties of performed Monte Carlo calculations is given in the text.

Table 5

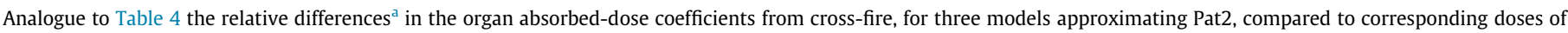
Pat2 (five sets of activity data were used).

\begin{tabular}{|c|c|c|c|c|c|c|c|c|c|}
\hline & \multicolumn{3}{|l|}{ RCP-AM } & \multicolumn{3}{|c|}{ P-RCP-AM } & \multicolumn{3}{|l|}{ Pat2M } \\
\hline & Mean & Min & Max & Mean & Min & Max & Mean & Min & Max \\
\hline Active bone marrow & $0.7 \%$ & $-2.1 \%$ & $6.4 \%$ & $-1.3 \%$ & $-4.3 \%$ & $4.3 \%$ & $17.9 \%$ & $14.1 \%$ & $26.3 \%$ \\
\hline Colon wall & $-15.5 \%$ & $-17.4 \%$ & $-13.8 \%$ & $-13.9 \%$ & $-16.4 \%$ & $-12.0 \%$ & $10.2 \%$ & $5.9 \%$ & $12.4 \%$ \\
\hline Lungs & $-28.0 \%$ & $-33.5 \%$ & $-25.1 \%$ & $-26.5 \%$ & $-31.5 \%$ & $-23.9 \%$ & $-7.2 \%$ & $-11.0 \%$ & $-4.7 \%$ \\
\hline Stomach wall ${ }^{\mathrm{b}}$ & $-29.8 \%$ & $-31.6 \%$ & $-24.7 \%$ & $-28.1 \%$ & $-29.9 \%$ & $-22.9 \%$ & $-6.7 \%$ & $-8.9 \%$ & $-0.8 \%$ \\
\hline Breast, total & $-7.0 \%$ & $-9.7 \%$ & $-3.2 \%$ & $-3.5 \%$ & $-6.7 \%$ & $1.4 \%$ & $19.2 \%$ & $13.2 \%$ & $28.0 \%$ \\
\hline Urinary bladder wall & $-0.9 \%$ & $-1.1 \%$ & $-0.5 \%$ & $-1.0 \%$ & $-1.2 \%$ & $-0.5 \%$ & $-0.4 \%$ & $-0.5 \%$ & $-0.2 \%$ \\
\hline Oesophagus & $-4.7 \%$ & $-6.5 \%$ & $-2.8 \%$ & $-6.9 \%$ & $-8.3 \%$ & $-5.4 \%$ & $17.1 \%$ & $14.0 \%$ & $19.0 \%$ \\
\hline Liver $^{\mathrm{b}}$ & $-34.4 \%$ & $-35.2 \%$ & $-34.0 \%$ & $-32.8 \%$ & $-33.4 \%$ & $-32.4 \%$ & $-10.0 \%$ & $-10.8 \%$ & $-9.4 \%$ \\
\hline Thyroid $^{\mathrm{b}}$ & $-7.5 \%$ & $-11.9 \%$ & $-5.4 \%$ & $-7.0 \%$ & $-11.1 \%$ & $-5.1 \%$ & $17.5 \%$ & $14.5 \%$ & $18.9 \%$ \\
\hline Brain & $-13.4 \%$ & $-14.4 \%$ & $-13.0 \%$ & $-12.4 \%$ & $-12.4 \%$ & $-12.3 \%$ & $6.7 \%$ & $5.7 \%$ & $9.2 \%$ \\
\hline Salivary glands ${ }^{\mathrm{b}}$ & $-42.1 \%$ & $-42.6 \%$ & $-41.6 \%$ & $-39.7 \%$ & $-40.9 \%$ & $-38.1 \%$ & $-16.3 \%$ & $-17.9 \%$ & $-13.9 \%$ \\
\hline Skin, total & $-15.5 \%$ & $-16.9 \%$ & $-14.5 \%$ & $-16.3 \%$ & $-17.4 \%$ & $-15.6 \%$ & $-0.9 \%$ & $-1.6 \%$ & $0.3 \%$ \\
\hline Adrenals & $-25.4 \%$ & $-29.4 \%$ & $-19.8 \%$ & $-24.7 \%$ & $-28.7 \%$ & $-19.0 \%$ & $-2.8 \%$ & $-8.0 \%$ & $3.6 \%$ \\
\hline Extrathoracic airways (ET) & $-25.5 \%$ & $-29.9 \%$ & $-23.3 \%$ & $-22.7 \%$ & $-26.3 \%$ & $-21.0 \%$ & $-4.8 \%$ & $-6.2 \%$ & $-4.1 \%$ \\
\hline Gall bladder wall & $-8.2 \%$ & $-13.1 \%$ & $-3.6 \%$ & $-11.0 \%$ & $-15.2 \%$ & $-7.5 \%$ & $19.4 \%$ & $9.9 \%$ & $23.8 \%$ \\
\hline Heart $^{\mathrm{b}}$ & $-5.1 \%$ & $-13.5 \%$ & $-0.3 \%$ & $-5.8 \%$ & $-14.7 \%$ & $-0.5 \%$ & $18.5 \%$ & $8.3 \%$ & $24.5 \%$ \\
\hline Kidneys $^{\mathrm{b}}$ & $-38.0 \%$ & $-38.9 \%$ & $-35.8 \%$ & $-36.0 \%$ & $-36.8 \%$ & $-33.7 \%$ & $-14.6 \%$ & $-16.0 \%$ & $-11.3 \%$ \\
\hline Pancreas ${ }^{\mathrm{b}}$ & $-38.1 \%$ & $-38.7 \%$ & $-37.1 \%$ & $-38.1 \%$ & $-38.9 \%$ & $-37.3 \%$ & $-16.9 \%$ & $-18.2 \%$ & $-15.8 \%$ \\
\hline Small intestine wall & $-29.4 \%$ & $-33.8 \%$ & $-24.5 \%$ & $-34.8 \%$ & $-38.2 \%$ & $-30.9 \%$ & $-18.1 \%$ & $-22.9 \%$ & $-13.6 \%$ \\
\hline Spleen $^{\mathrm{b}}$ & $-21.9 \%$ & $-26.3 \%$ & $-18.0 \%$ & $-20.1 \%$ & $-25.1 \%$ & $-16.0 \%$ & $3.3 \%$ & $-4.0 \%$ & $7.8 \%$ \\
\hline Thymus & $-26.9 \%$ & $-29.5 \%$ & $-23.1 \%$ & $-27.5 \%$ & $-29.9 \%$ & $-23.8 \%$ & $-12.7 \%$ & $-16.3 \%$ & $-8.3 \%$ \\
\hline Spinal cord & $-25.3 \%$ & $-28.4 \%$ & $-22.2 \%$ & $-24.1 \%$ & $-26.9 \%$ & $-21.3 \%$ & $-7.9 \%$ & $-9.4 \%$ & $-5.4 \%$ \\
\hline Skeleton, total & $-8.9 \%$ & $-10.9 \%$ & $-6.5 \%$ & $-9.2 \%$ & $-11.2 \%$ & $-6.7 \%$ & $7.3 \%$ & $4.6 \%$ & $12.0 \%$ \\
\hline Muscle tissue & $-24.7 \%$ & $-27.7 \%$ & $-22.2 \%$ & $-20.2 \%$ & $-22.3 \%$ & $-18.8 \%$ & $-4.2 \%$ & $-5.0 \%$ & $-3.5 \%$ \\
\hline Eyes & $-27.1 \%$ & $-32.2 \%$ & $-24.4 \%$ & $-22.7 \%$ & $-27.6 \%$ & $-20.2 \%$ & $-2.6 \%$ & $-5.6 \%$ & $-1.0 \%$ \\
\hline Eye lenses & $-28.4 \%$ & $-33.7 \%$ & $-25.6 \%$ & $-26.2 \%$ & $-33.3 \%$ & $-22.4 \%$ & $-12.4 \%$ & $-17.6 \%$ & $-9.5 \%$ \\
\hline
\end{tabular}

a The differences are expressed relative to the absorbed-dose coefficients from cross-fire for Pat2, since these are the patient-specific ones.

b These are the source organs for ${ }^{18} \mathrm{~F}-\mathrm{FSPG}$. 

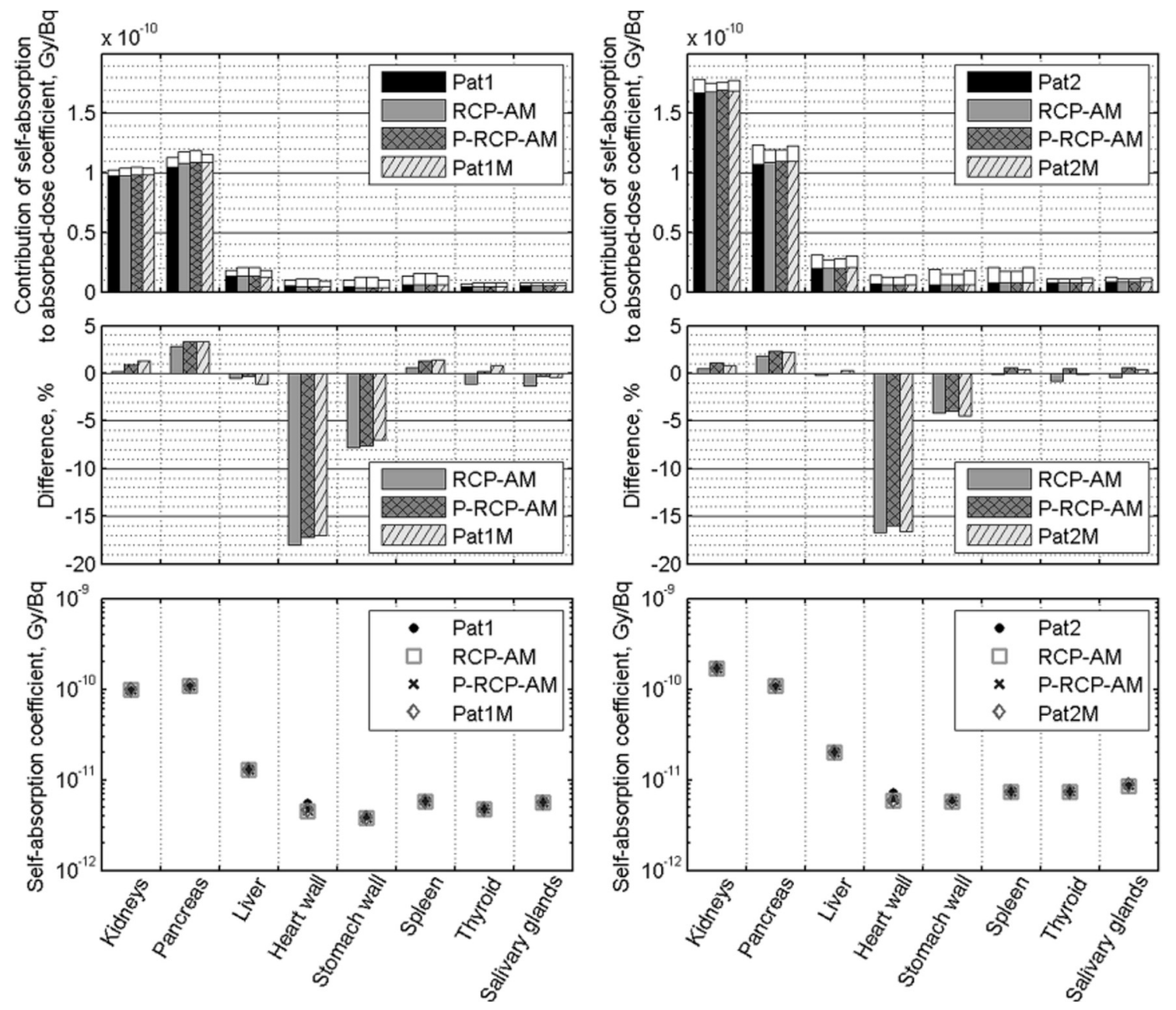

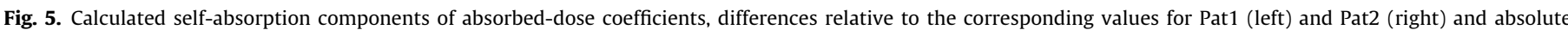

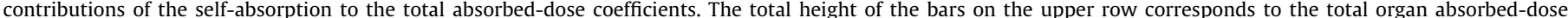

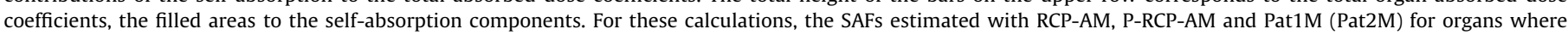
source is equal to target were adjusted according to the masses of the target patient Pat1 (Pat2).

sistent with the corresponding values in Pat2 within 5.6\%. For liver and salivary glands the calculated relative cross-fire dose differences between Pat2M and Pat2 are only one third of those between reference phantoms and Pat2, for skin and eyes only one tenth. For the cross-fire absorbed-dose coefficients for active bone marrow, breast, oesophagus, thyroid, gall-bladder wall and heart wall better agreement was observed between Pat2 and reference phantoms, though. Absorbed-dose coefficients from cross-fire for all organs and tissues of Pat2M considered, except the active bone marrow and breast, are consistent with the ones of Pat2 within 25.0\% (Table 5). Despite the gross gender-specific differences between Pat2 and Pat2M, the difference in the organ absorbed-dose coefficients from cross-fire were still held to $<25.0 \%$ for most organs. The gender-specific organs cannot be compared in this case.

\section{Discussion}

The voxelised versions $\left(1 \times 1 \times 1 \mathrm{~mm}^{3}\right.$ voxel size $)$ of the polygon-surface models were employed for the Monte Carlo calculations in this work. This potentially introduces the limitation of the voxelised models to realistically represent very small $(<1 \mathrm{~mm}$ in at least one dimension) anatomical structures. Nonetheless, the voxel size used is notably smaller than in the models seg- mented directly from the tomographic images (see e.g. Table 2) and it was sufficient for retaining the anatomical realism of most of the organs. The voxelisation can be theoretically done with even finer voxel sizes if accurate dosimetry is needed in specific cases, e.g. for ET, oral mucosa, eye lenses, basal cells of the epidermis etc. The limiting factors in this case are the available memory to store the phantom and the increasing calculation time of the Monte Carlo simulations when decreasing the voxel size.

Self-absorption is the main contribution to the organ absorbeddose coefficients for most source organs, especially kidneys, pancreas, salivary glands, liver and thyroid. Fig. 5 shows that the differences in the self-absorption are mostly eliminated by applying a mass-correction to the SAFs reflecting the masses of a target patient. Higher differences in the self-absorption dose coefficients between the target patient-specific models Pat 1 and Pat 2 and the phantoms approximating them were observed for heart wall (16-18\%) and stomach wall (4-8\%). The mass-scaling of the SAFs applied might have limitations for walled organs. Additionally, Pat1 and Pat2 do not have heart wall segmented separately, but only the whole heart instead. This could be another reason for the differences observed for the heart wall, since the selfabsorption in the whole heart is expected to be higher than that in the heart wall only. For some source organs, e.g. stomach wall 

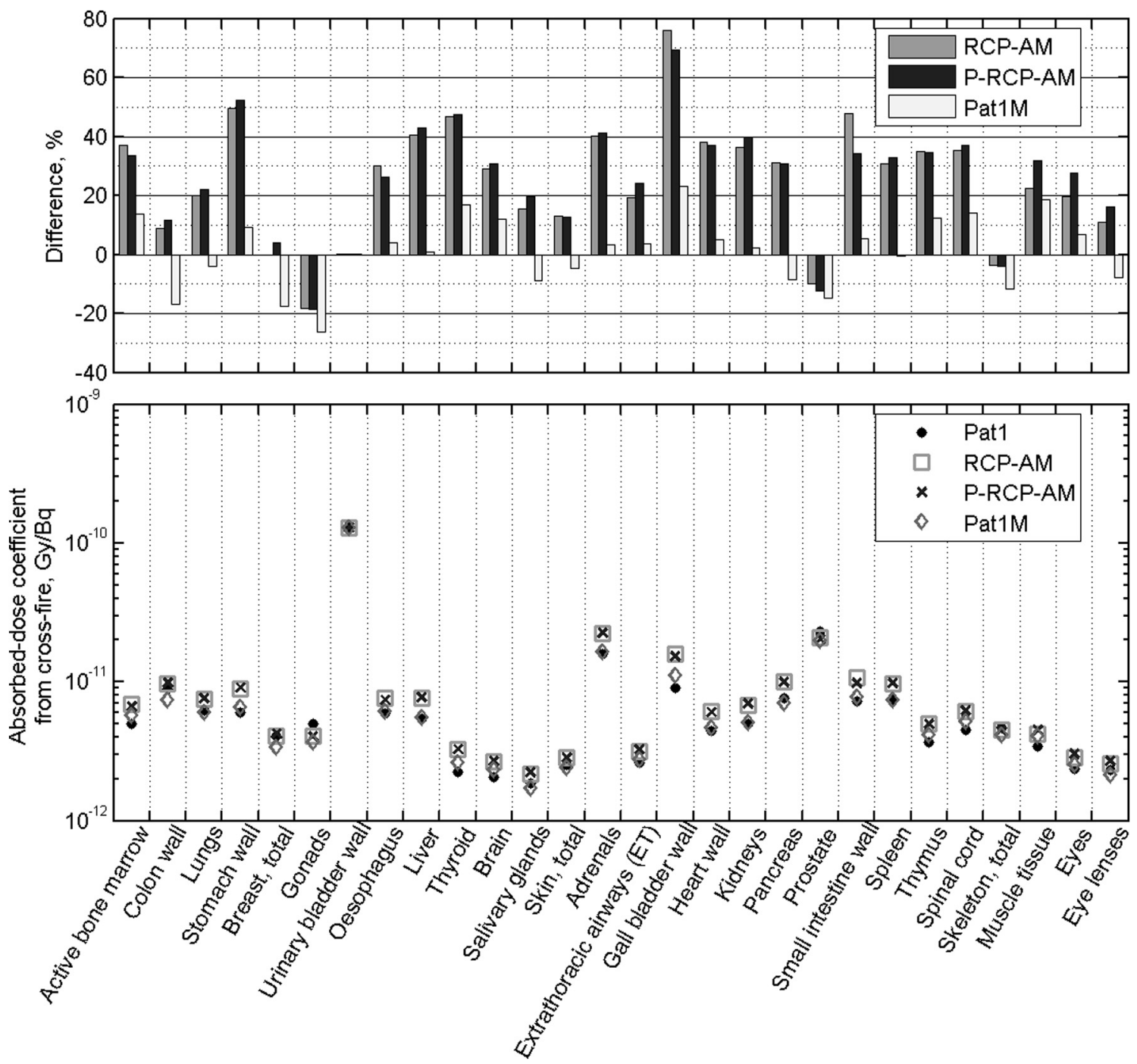

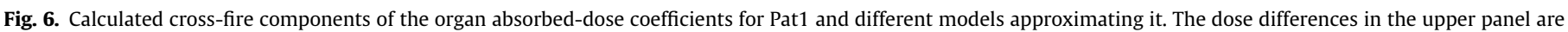
given relative to Pat1 (see also footnote b of Table 4).

and spleen, the absolute contributions to the absorbed-dose coefficients from the cross-fire were also substantial due to high activities of some neighbouring source organs.

High contribution to the absorbed-dose coefficients by the cross-fire from blood was observed for some organs, e.g. lungs, oesophagus, extrathoracic airways, thymus, eyes and eye lenses. The differences in the cross-fire from blood cannot be reduced by phantom adjustments. The cross-fire contribution from blood depends on how blood is attributed to different body organs and tissues in the Monte Carlo calculations. Since individual blood volumes and individual blood contributions in different organs and tissues are usually not available, the cross-fire from blood is a source of uncertainty of the absorbed-dose estimation and requires further careful considerations that go beyond the scope of this work.

We showed the feasibility of reducing the differences between the patient-specific cross-fire and the cross-fire estimated for adjusted reference models for most organs. Nonetheless, the results might differ for other radiopharmaceuticals, which have other source regions than ${ }^{18} \mathrm{~F}-\mathrm{FSPG}$. Comprehensive conclusions could be drawn if we compared the SAFs evaluated for all source-target combinations of the phantoms and different energies of the emitted $\beta$-particles (not only those corresponding to the $\beta$-spectrum of $\left.{ }^{18} \mathrm{~F}\right)$. However, such a comparison is practically very difficult because there are too many source-target combinations. Additionally, it is not generally obvious how the differences in the SAFs are propagated to the differences in the resulting organ absorbed-dose coefficients, since this also depends on the absolute values of SAFs and the absolute values of time-integrated activity coefficients. A considerable methodological limitation of our work is the usage of only two target patient-specific phantoms. We selected Pat 1 and Pat2 as the biggest and the smallest models in the HMGU library, respectively. They represent individuals with a pronounced difference in the BMI $\left(31.8 \mathrm{~kg} / \mathrm{m}^{2}\right.$ and $19.2 \mathrm{~kg} / \mathrm{m}^{2}$ for Pat1 and Pat2, respectively). Pat1 and Pat2 also have unique and unrelated organ topology. Relatively simple adjustments without matching the locations and masses of individual organs to those of Pat 1 and Pat 2 were applied in this work. Consequently, the cross-fire dose coefficients between the two fictitious target patients considered and the adjusted polygon-surface reference phantom P-RCP-AM were consistent for most organs within $25 \%$. Employing reference phantoms without any adjustment resulted in estimations of patient cross-fire dose coefficients within approximately 50\% relative uncertainty for the majority of target regions. However, the achieved improvement in the accuracy of organ dose coefficients might not apply to other individuals. To capture the 

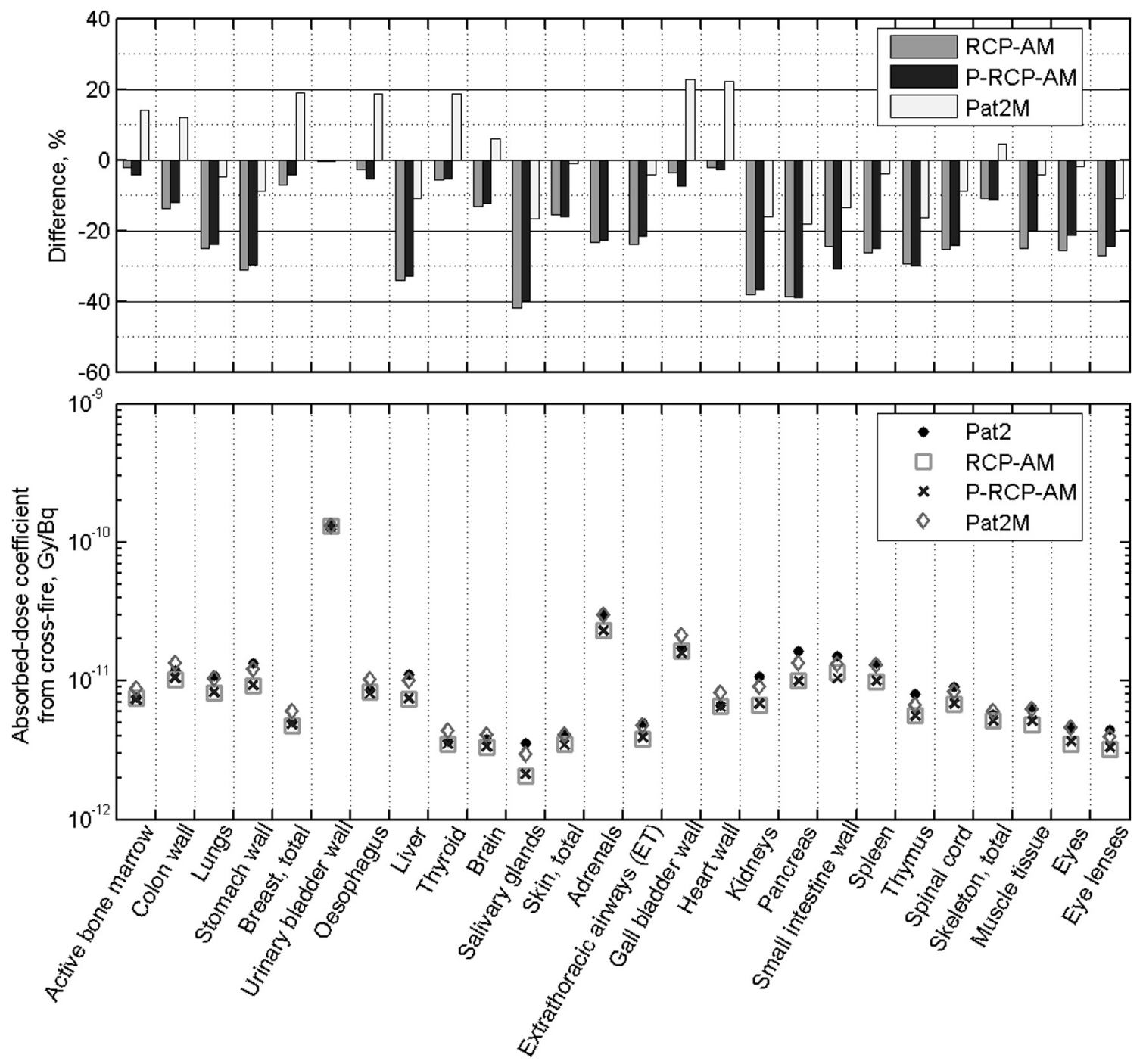

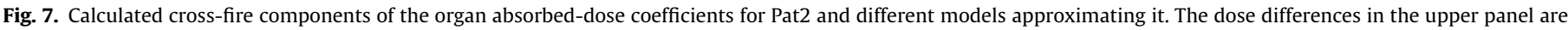
given relative to Pat2 (see also footnote b of Table 4).

whole range of inter-individual anatomic variability, more patientspecific phantoms would be required. The female fictitious target patient Pat 2 was represented by male anatomy of the adjusted PRCP-AM. Thus gender-specific organs of Pat 2 were not considered. This is a limitation of the presented work. The results of our test study showed the feasibility of reducing the uncertainties in the cross-fire absorbed-dose coefficients for both patient-specific models employed, despite them being segmented from individuals with unrelated anatomy and pronounced difference in the BMI.

For a convenient application of the proposed method the dimensions to be adjusted should be relatively easy to obtain for individual patients. The set of dimensions adjusted in this work was selected accordingly. For constructing Pat1M and Pat2M individual organ masses and positions were not adjusted to match the corresponding values of the patient-specific phantoms Pat1 and Pat2. The employed mass-correction of the SAFs is phantomindependent and requires only the masses of the target = source regions. The clinical quantification of the absolute activities in the source regions - which in any case needs to be determined to yield accurate personalised self-absorption doses - implicitly includes the estimation of the source region masses as part of its procedure. The absolute activities are calculated via the multiplication of the activity concentrations, determined from the PET images, and the source region volumes, obtained from the registered CT images.

Patient-specific dosimetry potentially provides more accurate dose values compared to our proposed approach. The former can be implemented in two ways: a direct dosimetry based on patient CT images or a conversion of patient tomographic images into a voxelized patient-specific phantom, followed by the corresponding dosimetry. However, both patient-specific options require the contouring of all regions of interest. This is often difficult in clinical conditions, especially with a lack of automated segmentation tools. Additionally, patient-specific dosimetry requires individual CT or magnetic resonance images of sufficiently large body coverage and adequate resolution to realistically represent even small organs and tissues. Such tomographic data are not always available. Although patient-specific dosimetry on patient tomographic images is considered the most accurate dosimetry option, the abovementioned factors often limit its application in medical practice. The proposed approach can be useful in those scenarios where the patient-specific dosimetry is not feasible. Our study showed the potential to improve the dosimetry in nuclear medicine by adjusting P-RCP-AM to the dimensions of a patient. This approach requires less effort than would be needed were we to segment a patient-specific model. An alternative approach could be to select 
the phantom, which agrees best with the specific patient under consideration, from the already existing large libraries $[8,9,11,12$, 14,15,17-19]. However, since most of these libraries were constructed based on variations in height and weight as primary parameters, the approach chosen here can help adjusting the selected phantom to the dimensions of the specific patient even better, without too much effort. The dimensions adjusted in our study include front-to back distance and width of the rib cage, height, distance between the iliac crest and clavicles. These dimensions could be also considered in selecting the phantom that best matches to the individual patient. This might further reduce the differences in the estimated doses.

\section{Conclusions}

To evaluate the feasibility of reducing the uncertainty in the estimated doses in nuclear medicine caused by anatomical difference between the patient-specific and the reference phantom, we adjusted the polygon-surface ICRP adult male reference phantom to a set of external dimensions of two investigated individuals. They were represented by the voxel phantoms Visible Human (=Pat1) and Irene (=Pat2). Using the two resulting phantoms from the adjustment and five sets of activity data of a radiopharmaceutical, designated for PET/CT imaging, we studied which agreement in the computed doses can be achieved. The results of our pilot study showed the feasibility of reducing the uncertainty in the cross-fire component of the estimated dose coefficients by a relatively simple phantom adjustment; that is, $<25 \%$ of relative uncertainty in dose coefficients from cross-fire for most organs and tissues. Overall, the proposed approach provided reliable dose estimates for both tested patients despite the pronounced differences in their anatomy. To capture the full range of inter-individual variability more patients would be required. We believe the proposed approach could be a good trade-off balancing the effort to be expended and the accuracy to be achieved. It has the potential to be directly applicable in medical practice, in contrast to the less viable segmentation of a patient-specific phantom. The level of personalisation of an employed anatomical model should depend on the application and on the available resources. Our results suggest that the proposed approach could be reliable enough for most situations involving low to moderate doses (i.e. outside the therapy realm). Better agreement in cross-fire could also be achieved by using a phantom selected from a collection of diversified models which best matches the external dimensions of the patient.

\section{Conflict of interest statement}

This work was supported by the German Bundesministerium für Bildung und Forschung [grant number 02NUK026]. The authors declare no additional conflicts of interest.

\section{Acknowledgments}

This work was supported by the German Bundesministerium für Bildung und Forschung [grant number 02NUK026].

We thank Prof. Markus Schwaiger, PD Stephan G. Nekolla and Frank Philipp Graner from the Department of Nuclear Medicine of Technische Universität München for providing the activity data of the radiopharmaceutical used in this work.

\section{References}

[1] ICRP, Adult reference computational phantoms, International Commission on Radiological Protection, Oxford, ICRP Publication 110, 2009.
[2] Xu XG. An exponential growth of computational phantom research in radiation protection, imaging, and radiotherapy: a review of the fifty-year history. Phys Med Biol 2014;59:R233-302.

[3] Bolch W, Lee C, Wayson M, Johnson P. Hybrid computational phantoms for medical dose reconstruction. Radiat Environ Biophys 2010:49:155-68.

[4] Petoussi-Henss N, Zankl M, Fill U, Regulla D. The GSF family of voxel phantoms. Phys Med Biol 2002;47:89-106.

[5] Fill U, Zankl M, Petoussi-Henss N, Siebert M, Regulla D. Adult female voxel models of different stature and photon conversion coefficients for radiation protection. Health Phys 2004;86:253-72.

[6] Zankl M, Fill U, Petoussi-Henss N, Regulla D. Organ dose conversion coefficients for external photon irradiation of male and female voxel models. Phys Med Biol 2002;47:2367-85.

[7] Schlattl H, Zankl M, Petoussi-Henss N. Organ dose conversion coefficients for voxel models of the reference male and female from idealized photon exposures. Phys Med Biol 2007:52:2123-45.

[8] Lee C, Williams JL, Lee C, Bolch WE. The UF series of tomographic computational phantoms of pediatric patients. Med Phys 2005;32:3537-48.

[9] Lee C, Lee C, Williams JL, Bolch WE. Whole-body voxel phantoms of paediatric patients - UF series B. Phys Med Biol 2006;51:4649-61.

[10] Na YH, Zhang BQ, Zhang JY, Caracappa PF, Xu XG. Deformable adult human phantoms for radiation protection dosimetry: anthropometric data representing size distributions of adult worker populations and software algorithms. Phys Med Biol 2010;55:3789-811.

[11] Broggio D, Beurrier J, Bremaud M, Desbrée A, Farah J, Huet C, et al. Construction of an extended library of adult male 3D models: rationale and results. Phys Med Biol 2011;56:7659-92.

[12] Cassola VF, Milian FM, Kramer R, de Oliveira Lira CAB, Khoury HJ. Standing adult human phantoms based on 10th, 50th and 90th mass and height percentiles of male and female Caucasian populations. Phys Med Biol 2011;56:3749.

[13] Ding A, Mille M, Liu T, Caracappa PF, Xu XG. Extension of RPI-adult male and female computational phantoms to obese patients and a Monte Carlo study of the effect on CT imaging dose. Phys Med Biol 2012;57:2441-76.

[14] Segars WP, Bond J, Frush J, Hon S, Eckersley C, Williams CH, et al. Population of anatomically variable 4D XCAT adult phantoms for imaging research and optimization. Med Phys 2013:40:043701.

[15] Segars WP, Norris H, Sturgeon GM, Zhang Y, Bond J, Minhas A, et al. The development of a population of 4D pediatric XCAT phantoms for imaging research and optimization. Med Phys 2015:42:4719-26.

[16] Norris H, Zhang Y, Bond J, Sturgeon GM, Minhas A, Tward DJ, et al. A set of 4D pediatric XCAT reference phantoms for multimodality research. Med Phys 2014;41:033701.

[17] Geyer AM, O'Reilly S, Lee C, Long DJ, Bolch WE. The UF/NCI family of hybrid computational phantoms representing the current US population of male and female children, adolescents, and adults-application to CT dosimetry. Phys Med Biol 2014:59:5225-42.

[18] Lee C, Lodwick D, Hurtado J, Pafundi D, Lwilliams J, Bolch WE. The UF family of reference hybrid phantoms for computational radiation dosimetry. Phys Med Biol 2010;55:339-63.

[19] Chen Y, Qiu R, Li C, Wu Z, Li J. Construction of Chinese adult male phantom library and its application in the virtual calibration of in vivo measurement. Phys Med Biol 2016;61:2124-44.

[20] Zhang Y, Li X, Segars WP, Samei E. Organ doses, effective doses, and risk indices in adult CT: comparison of four types of reference phantoms across different examination protocols. Med Phys 2012;39:3404-23.

[21] Segars WP, Sturgeon G, Mendonca S, Grimes J, Tsui BM. 4D XCAT phantom for multimodality imaging research. Med Phys 2010:37:4902-15.

[22] Smith T, Petoussi-Henss N, Zankl M. Comparison of internal radiation doses estimated by MIRD and voxel techniques for a 'family' of phantoms. Eur J Nucl Med 2000;27:1387-98.

[23] Zankl M, Petoussi-Henss N, Fill U, Regulla D. The application of voxel phantoms to the internal dosimetry of radionuclides. Radiat Prot Dosimetry 2003; $105: 539-48$.

[24] Petoussi-Henss N, Bolch WE, Zankl M, Sgouros G, Wessels B. Patient-specific scaling of reference S-values for cross-organ radionuclide S-values: what is appropriate? Radiat Prot Dosimetry 2007;127:192-6.

[25] Stabin MG. Fundamentals of nuclear medicine dosimetry. Springer Science \& Business Media; 2008.

[26] ICRP, The ICRP computational framework for internal dose assessment for reference adults: specific absorbed fractions, International Commission of Radiological Protection, Oxford, UK, ICRP Publication 133, 2016.

[27] Snyder WS, Ford MR, Warner GG, Watson EE, "S" absorbed dose per unit cumulated activity for selected radionuclides and organs, Society of Nuclear Medicine, New York, NY, MIRD Pamphlet 11, Revised, 1975.

[28] Whalen S, Lee C, Williams JL, Bolch WE. Anthropometric approaches and their uncertainties to assigning computational phantoms to individual patients in pediatric dosimetry studies. Phys Med Biol 2008;53:453-71.

[29] Caon M, Bibbo G, Pattison J. Monte Carlo calculated effective dose to teenage girls from Computed Tomography examinations. Radiat Prot Dosimetry 2000;90:445-8.

[30] Yeom YS, Han MC, Kim CH, Jeong JH. Conversion of ICRP male reference phantom to polygon-surface phantom. Phys Med Biol 2013;58:6985-7007.

[31] Nguyen TT, Yeom YS, Kim HS, Wang ZJ, Han MC, Kim CH, et al. Incorporation of detailed eye model into polygon-mesh versions of ICRP-110 reference phantoms. Phys Med Biol 2015;60:8695-707. 
[32] Yeom YS, Kim HS, Nguyen TT, Choi C, Han MC, Kim CH, et al. New smallintestine modeling method for surface-based computational human phantoms. J Radiol Prot 2016;36:230-45.

[33] Schlattl H, Zankl M, Becker J, Hoeschen C. Dose conversion coefficients for CT examinations of adults with automatic tube current modulation. Phys Med Biol 2010;55:6243-61.

[34] Xu XG, Chao TC, Bozkurt A. VIP-MAN: an image-based whole-body adult male model constructed from color photographs of the Visible Human Project for multi-particle Monte Carlo calculations. Health Phys 2000;78:476-86.

[35] Kawrakow I, Rogers DWO, The EGSnrc code system: Monte Carlo simulation of electron and photon transport, National Research Council of Canada (NRCC), Ottawa, PIRS Report 701, 2003.

[36] Archambault JP, Mainegra-Hing E. Comparison between EGSnrc, Geant4, MCNP5 and Penelope for mono-energetic electron beams. Phys Med Biol 2015;60:4951-62.

[37] ICRP, Conversion Coefficients for Radiological Protection Quantities for External Radiation Exposures, International Commission of Radiological Protection, Oxford, UK, ICRP Publication 116, 2010.

[38] Hadid L, Desbrée A, Schlattl H, Franck D, Blanchardon E, Zankl M. Application of the ICRP/ICRU reference computational phantoms to internal dosimetry: calculation of specific absorbed fractions of energy for photons and electrons. Phys Med Biol 2010;55:3631-41.

[39] Nooruddin FS, Turk G. Simplification and repair of polygonal models using volumetric techniques. IEEE Trans Vis Comput Graphics 2003;9:191-205.

[40] Zvereva A, Petoussi-Henss N, Li WB, Schlattl H, Oeh U, Zankl M, et al. Effect of blood activity on dosimetric calculations for radiopharmaceuticals. Phys Med Biol 2016;61:7688-703.

[41] Nadler SB, Hidalgo JH, Bloch T. Prediction of blood volume in normal human adults. Surgery 1962;51:224-32.
42] ICRU, Photon, electron, proton and neutron interaction data for body tissues, International Commission on Radiation Units and Measurements, Bethesda, MD, ICRU Report 46, 1992

[43] ICRP, Basic anatomical and physiological data for use in radiologica protection: reference values, Pergamon, Oxford, ICRP Publication 89, 2002.

[44] Bolch WE, Eckerman KF, Sgouros G, Thomas SR. MIRD pamphlet No. 21: a generalized schema for radiopharmaceutical dosimetry-standardization of nomenclature. J Nucl Med 2009;50:477-84.

[45] Zankl M, Schlattl H, Petoussi-Henss N, Hoeschen C. Electron specific absorbed fractions for the adult male and female ICRP/ICRU reference computational phantoms. Phys Med Biol 2012;57:4501.

[46] ICRP, Limits for intakes of radionuclides by workers. Part 1, Pergamon Press, Oxford, UK, ICRP Publication 30, 1979.

[47] Cristy M, Eckerman KF, Specific absorbed fractions of energy at various ages from internal photon sources, Part I: Methods, Oak Ridge National Laboratory, Oak Ridge, TN, ORNL Report TM-8381/V1, 1987.

[48] ICRP, Nuclear decay data for dosimetric calculations, Internationa Commission of Radiological Protection, Oxford, ICRP Publication 107, 2008.

[49] Smith T, Veall N, Wootton R. Bladder wall dose from administered radiopharmaceuticals: the effects of variations in urine flow rate, voiding interval and initial bladder content. Radiat Prot Dosimetry 1982;2:183-9.

[50] Thomas SR, Stabin MG, Chen CT, Samaratunga RC, Pamphlet MIRD. No. 14 revised: a dynamic urinary bladder model for radiation dose calculations. J Nucl Med 1999;40:102S-23S.

[51] Andersson M, Minarik D, Johansson L, Mattsson S, Leide-Svegborn S. Improved estimates of the radiation absorbed dose to the urinary bladder wall. Phys Med Biol 2014:59:2173-82. 